\title{
Effect of instructional environment on physics students' representational skills
}

\author{
Patrick B. Kohl and Noah D. Finkelstein \\ Department of Physics, University of Colorado at Boulder, Boulder, Colorado 80309, USA
}

(Received 6 September 2005; published 20 January 2006)

\begin{abstract}
In a recent study we showed that physics students' problem-solving performance can depend strongly on problem representation, and that giving students a choice of problem representation can have a significant impact on their performance [P. B. Kohl and N. D. Finklestein, Phys. Rev. ST. Phys. Educ. Res. 1, 010104 (2005)] In this paper, we continue that study in an attempt to separate the effect of instructional technique from the effect of content area. We determine that students in a reform-style introductory physics course are learning a broader set of representational skills than those in a more traditional course. We also analyze the representations used in each course studied and find that the reformed course makes use of a richer set of representations than the traditional course and also makes more frequent use of multiple representations. We infer that this difference in instruction is the source of the broader student skills. These results provide insight into how macrolevel features of a course can influence student skills, complementary to the microlevel picture provided by the first study.
\end{abstract}

DOI: 10.1103/PhysRevSTPER.2.010102

PACS number(s): 01.40.Fk

\section{INTRODUCTION}

Our previous work $^{1}$ showed that physics students' problem-solving success depends on the representation of the problem, corroborating other work in physics education research (PER). ${ }^{2}$ That is, whether one represents a physics problem in terms of words, equations, graphs, or pictures can have a significant impact on student performance on that problem. Our work follows a number of PER studies that have investigated student skills with particular representations of physics concepts and problems, ${ }^{2-5}$ and that have studied how best to explicitly teach students how to use multiple representations to solve physics problems. ${ }^{6-8}$

In our previous work, ${ }^{1}$ we also investigated whether providing students with a choice of representational format would help or harm their problem-solving performance. That is, we asked whether students are capable of determining which representations are most helpful to them on problems of a given topic. The results from our study were complicated: Student problem-solving performance often depended very strongly on whether or not they had a choice of problem format, but the strength and direction of the effect varied with the representation, the subject matter, and the instructor. Such a study considers students' metarepresentational skills, and asks what students know about representations. Studies on metarepresentational competence are relatively rare, but the topic is becoming increasingly popular. ${ }^{9-12}$

\section{Previous study-overview}

Our first study took place in two different large-lecture algebra-based introductory physics courses using the same textbook. ${ }^{13}$ Physics 201 covered mechanics and was taught by a reform-style instructor who made heavy use of concept tests, ${ }^{14}$ personal response systems ("clickers"), ${ }^{15}$ and wellintegrated lecture demonstrations. Physics 202 covered electromagnetism, optics, and atomic physics, and was taught by a traditional-style professor, though he used clicker questions to a limited extent. Neither professor is a researcher on this project. Note that these 201 and 202 courses were not insequence; that is, the students in the reformed 201 class were not members of the traditional 202 class, though they composed similar populations. The courses included weekly twohour recitation/lab sections. In each course, we gave students prerecitation homeworks that had four problems, one in each of four different representational formats (verbal, mathematical, graphical, and pictoral). We then gave these students a one-question quiz during their recitation section. This quiz was in one of four formats, and one-half to two-thirds of the sections were allowed to choose which quiz format they would receive without examining the questions ahead of time. The students in the rest of the sections received a quiz in a random format. This second group provided a control for the study on representation choice. This process was repeated later in each term for a different topic, for a total of four trials. The 201 quiz topics were springs and pendulums, while the 202 quiz topics were diffraction and spectroscopy.

In both courses, we found ample evidence that student problem-solving performance can (but does not necessarily) depend strongly on representation, strengthening results seen elsewhere.,16 This finding naturally leads to microlevel questions regarding when and how problem solving depends on representation. In some cases, a problem feature was present in one representation but was either not present or not as attractive in another representation. We also observed in interviews that student problem-solving strategies depend sensitively on problem representation, a result that we will consider in depth in a future paper.

The effect of providing a choice of problem formats was complex. In some cases giving students a choice of format resulted in significantly increased performance with respect to the random-format control sections. In other cases, the choice of formats resulted in significantly decreased performance. These choice/control differences were often quite strong, and also varied by topic area. A group that chose one format would do significantly better than the corresponding control group for one topic, and then would do significantly worse on the next quiz topic when working in the same 
TABLE I. Statistical significance of the quiz performance differences between the format choice and control groups in the traditional 202 and reform 201 sections. Numbers are $p$ values using a two-tailed binomial proportion test. $X$ denotes a $p$ value of greater than 0.10 . Bold indicates that the choice group had higher performance than the control group.

\begin{tabular}{lllll}
\hline \hline Quiz subject & Verbal & Math & Graphical & Pictoral \\
\hline 202 Diffraction & X & X & 0.04 & $\mathbf{0 . 0 3}$ \\
202 Spectroscopy & $\mathbf{0 . 0 0 2}$ & $\mathbf{0 . 0 0 0 1}$ & $\mathbf{0 . 0 0 0 4}$ & 0.001 \\
201 Springs & X & 0.09 & $\mathbf{X}$ & $\mathbf{0 . 0 7}$ \\
201 Pendulums & $\mathbf{X}$ & $\mathbf{X}$ & $\mathbf{X}$ & $\mathbf{X}$ \\
\hline \hline
\end{tabular}

format. The performance differences between choice and control groups are shown in Table I, reprinted from our previous paper. ${ }^{1}$ Each entry is the statistical significance $(p$ value, using a two-tailed binomial proportion test) of the difference between the performance of the choice and control groups for a given combination of quiz topic and quiz format. Bold entries indicate that the sections that had a choice of problem representation outperformed the control groups; unbolded indicates the opposite. An $X$ indicates a $p$ value greater than 0.10 . Notable features include the strength of many of the choice/control splits and the changing direction of the effect across topic. Especially interesting is the fact that the 202 course showed much stronger choice/control splits than the 201 course. This led to macrolevel questions: Was this qualitative difference in performance data a result of the different instructional style, the different content area, or some combination? Also, what was it about the difference in content or instruction that was associated with the difference in performance?

We shall address these questions in two parts. As we noted before, these courses were taught by different professors and covered different material, and so the differences observed could conceivably be explained by differences in instruction, differences in content, or some combination. Our previous hypothesis was that the much different approach of the reformed 201 course resulted in students having a broader set of representational skills. Thus, whether or not the students received their preferred representation (that is, the representation they would have chosen given the opportunity) made less of a difference (positive or negative) in performance. The first part of this paper begins to test our hypothesis by separating out the effect of instruction from the effect of content. We repeated the Physics 202 study in the spring of 2005, when the course was taught by the reform-style professor who had been in charge of the 201 course in the earlier study. We predicted that given the same quizzes, the choice/control splits would be much weaker than they were in the original study with the traditional 202 professor. ${ }^{17}$ This prediction held true, leading to the second part of the paper, in which we analyze the specific differences in representation use in these classes in lectures, exams, and homeworks. The results allow us to conclude that a pervasive use of multiple representations in a physics course can support students learning a broader set of representational skills than students in a representationally sparse environment.

\section{STUDY: SEPARATING THE EFFECTS OF CONTENT AND INSTRUCTION}

\section{A. Methods: Study homeworks and quizzes}

The study was conducted in the same way, using the same materials, as the 202 study in the earlier work (see the brief recap above or Ref. 1). In Fig. 1 we see two example homework problems on atomic physics, in graphical and pictorial formats. In Fig. 2, we see an example of the verbal and pictorial format spectroscopy quizzes; the problem statements and one of the distractors are shown for each format. Note that in all quizzes, the statements and distractors mapped from one quiz representation to the next. The homework problems could not be made completely isomorphic since the students were assigned all of them, but they were

\section{Question 3 - Graphical \\ An electron in a Bohr hydrogen atom jumps from the $n=3$ orbit to the $n=2$ orbit. The following graphs show the orbit radius $r$ as a function of the orbit number $\mathrm{n}$. Choose the graph that best represents the relative locations of the electron orbits.}

A)

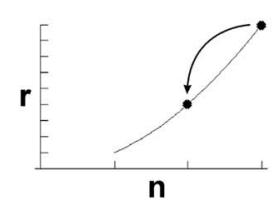

C)

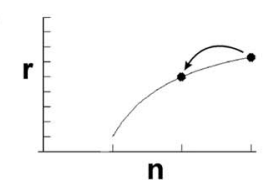

E) None of these.
B)

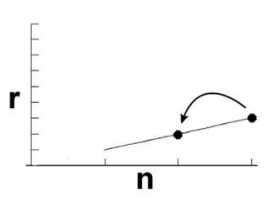

D)

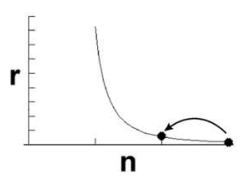

Question 4 - Pictorial

An electron in a Bohr hydrogen atom jumps from the $n=3$ orbit to the $n=1$ orbit. Choose the picture that best represents the relative locations of the electron orbits.

A)

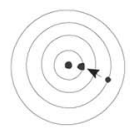

C)

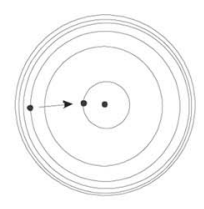

E) None of these.
B)

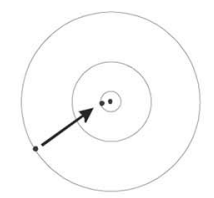

D)

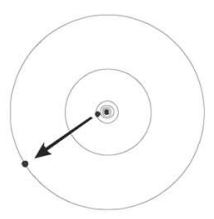

FIG. 1. Nearly isomorphic homework problems (in graphical and pictoral/ diagrammatic formats) regarding Bohr-model electron orbit radii. 
Spectroscopy Problem - Pictorial Format

The Balmer series of spectral lines is shown below, as seen through a spectrometer:

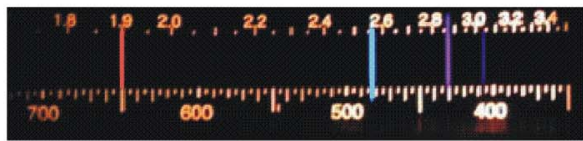

Now suppose we are in a world where electric charges are weaker, so the electron is not held as tightly by the nucleus and the ionization energy is $13 \mathrm{eV}$ instead of $13.6 \mathrm{eV}$. Choose the picture that best represents what the new spectrum would look like.
Spectroscopy Problem - Verbal Format

Consider the Balmer series of spectral lines from hydrogen gas. Now suppose we are in a world where electric charges are weaker, so the electron is not held as tightly by the nucleus. This means that the ionization energy for the electron will be smaller. What will happen to the Balmer lines that we see?

FIG. 2. (Color) Problem statement and second answer choice for the verbal and pictoral format quizzes. The other distractors (not shown) match up between the formats as well.

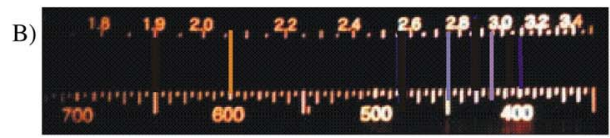

B) The spectral lines will all shift to shorter wavelengths (toward the bluer colors).

designed to be as similar as was reasonable. For instance, some problems asked students how the radius of a Bohrmodel electron's orbit varied with the orbital number. The different questions would be the same except for the representation and for which particular transition $(n=3$ to $n=2$, for example) was under consideration.

\section{B. Data}

In Table II, we see the performance of the reformed Physics 202 students on the pre-recitation homeworks. Notably, the fractional and statistical differences between the graphical and pictorial questions on the Bohr-model homework (a key comparison in Ref. 1) are smaller than they were in the traditional 202 course $(0.55$ vs 0.48 instead of 0.77 vs 0.62 , significant at $p=0.05$ versus $p=0.006$ ).

In Table III, we see the performance of the students on the diffraction and spectroscopy recitation quizzes, sorted by representation and by whether the students were in a format choice or control group. Performance variation across representation was generally less statistically significant in this course than it was in the traditional 202 section, including both quizzes and homeworks. ${ }^{1}$ We note here that the traditional students noticeably outperformed the reform students on the 202 diffraction quiz. For the sake of replication, the exact questions that were designed for the traditional 202 course were given to the students in the reform 202 course. The two 202 courses placed different emphases on the different subtopics available, and the reform section spent very little time on double finite-width slit diffraction (the focus of this quiz problem). Student comments and performance suggest that most students treated this as a double infinitesimalwidth slit problem. One of the distractors is correct for such an interpretation of the problem, and student selection of this distractor is noted in the (dist) line of Table III (where performance is noticeably higher). Because of the different emphases on specific content, comparisons of absolute performances across courses are not likely to be valid, and so we focus on relative student performance across different representations and across choice and control groups. ${ }^{18}$

In Table IV, we show the statistical significances ( $p$ values using a two-tailed binomial proportion test) of the differences between the choice and control groups on the different topics and formats. Note that these data are essentially the same if one considers the correct diffraction quiz answer to be the distractor mentioned above (the math $p$ value changes from 0.06 to 0.03 ; all others remain insignificant). When comparing these data to those in Table I, we see that they are very similar in character to the reformed 201 course, and much different in character than the traditional 202 course. This suggests that the choice/control splits (or lack thereof) are associated more closely with the instructor and course environment than with the content area. Thus, we analyze these environments in more detail with the goal of finding any differences in representational content and their uses.

\section{STUDY: ANALYSIS OF COURSE ENVIRONMENTS}

The second part of this study involves characterizing the representation use in each of the classes under consideration: the reform 201, the traditional 202, and the reform 202. These courses had many components, including lecture, lab/ recitation, exams, and homeworks. The homeworks included problems assigned and graded through a web-based automated system [CAPA (Ref. 19)], and long-answer handgraded problems. In comparing the courses, we judged the

TABLE II. Fraction of students answering a homework problem correctly, sorted by representational format and topic. Standard errors of the mean vary but are on the order of 0.02 .

\begin{tabular}{lcccc}
\hline \hline Reform course, Phys 202 & Verbal format & Mathematical & Graphical & Pictoral \\
\hline Diffraction/Interference HW $(N=332)$ & 0.44 & 0.36 & 0.39 & 0.46 \\
Spectroscopy/Bohr HW $(N=341)$ & 0.63 & 0.60 & 0.55 & 0.48 \\
\hline \hline
\end{tabular}


TABLE III. Quiz performance of students from the random-format recitation sections (left) and from the recitation sections that had a choice of formats (right). The number of students taking a quiz is in parentheses. The quiz topics are diffraction and spectroscopy. Standard errors of the mean range from 0.05 to 0.09 and are not shown. The last line indicates how many students chose a particular distractor on the diffraction quiz, as discussed in the text.

\begin{tabular}{lcccccccc}
\hline \hline \multirow{2}{*}{$\begin{array}{l}\text { Reform course } \\
\text { Phys 202 }\end{array}$} & \multicolumn{4}{c}{ Control (random format) group } & \multicolumn{4}{c}{ Choice group } \\
\cline { 2 - 10 } & Verbal & Math & Graphical & Pictoral & Verbal & Math & Graphical & Pictoral \\
\hline Diffraction & $0.19(46)$ & $0.35(46)$ & $0.14(46)$ & $0.18(44)$ & $0.15(16)$ & $0.57(34)$ & $0.13(37)$ & $0.21(77)$ \\
Spectroscopy & $0.59(46)$ & $0.39(46)$ & $0.57(42)$ & $0.54(46)$ & $0.41(17)$ & $0.32(25)$ & $0.49(37)$ & $0.52(89)$ \\
Diffraction (dist) & 0.33 & 0.45 & 0.44 & 0.45 & 0.26 & 0.22 & 0.41 & 0.32 \\
\hline \hline
\end{tabular}

labs/recitations and CAPA-based homeworks to have very similar representational characters. We thus focus our analysis on the lectures, exams, and long-answer homeworks. This approach provides two views of the class. We see how the use of physics representations was modeled for the students (the lectures), and how the students were held responsible for using physics representations (homeworks and exams).

\section{A. Methods}

\section{Analysis of course lectures}

We videotaped three to five lectures from each of the three courses, and then picked three lectures from each course for analysis. These lectures were chosen to span the material covered in the study quizzes.

In order to quantify the different uses of representations in the different lectures, we divided each tape into one-minute sections. ${ }^{20}$ For each segment, we noted which representations were used significantly according to the following rubric.

(i) Verbal: Writing sentences expressing an idea or concept on the board; presenting and explicitly referring to a slide with verbal-only content for the sake of the point at hand (words surrounding mathematics are not counted).

(ii) Mathematical: Writing equations; explicitly referring to equations for the sake of the point at hand; doing mathematics. Writing numerical data by themselves is not counted (for example, simply writing $h=6.636 \times 10^{-34} \mathrm{Js}$ does not count).

(iii) Graphical: Drawing or modifying a graph; explicitly referring to a graph for the sake of the point at hand.

(iv) Pictorial: Drawing or modifying a picture; explicitly referring to a picture for the sake of the point at hand.

(v) Physical demonstration: Carrying out a physical demonstration.

Note that for lectures, we have added the representational category "Physical demonstration." We also noted which intervals include clicker questions. Finally, any interval in which more than one representation was used was coded as a multiple representations interval (the Clicker category did not count for this purpose).

Because the professor is speaking during nearly every part of a lecture, we did not count spoken words towards the use of verbal representations. This is an example of a broader feature of this study: the privileged position of the verbal representation. Essentially every aspect of the course had some verbal component (even math problems include explanatory text), and so we necessarily have stricter standards as to what counts as verbal representation use compared to the other categories.

Once a lecture was coded, we calculated the fraction of the lecture that showed use of each of the representational categories. We then averaged across the three lectures from each class to obtain an average representational content for those courses' lectures. To test for interrater reliability, two individuals (the principal investigator and a researcher unrelated to this study) coded a subset of three lectures working from a written description of the coding standard. Results were extremely similar; the average difference between raters on each category was $1.3 \%$. With approximately 50 oneminute bins per lecture, this represents a typical coding difference of one bin per representation per lecture. This difference is very low, which we attribute to the length and detail of the written coding standard (much of which is shown above).

\section{Analysis of exams}

Each of the three courses considered issued three exams, not including the final. The final exam took place after all the recitation quizzes and homeworks used in the study, and thus is not included in the analysis. We quantified the fraction of each exam that could be described as verbal, mathematical, graphical, and pictorial in representation. We also quantified the fraction of each exam that explicitly required the use of multiple representations. The coding occurred as follows: On an exam, each subproblem is designated as having a verbal, mathematical, graphical, or pictorial component or some

TABLE IV. Statistical significance of the quiz performance differences between the format choice and control groups in the reformed 202 section. Numbers are $p$ values using a two-tailed binomial proportion test. $X$ denotes a $p$ value of greater than 0.10 . Bold indicates that the choice group had higher performance than the control group.

\begin{tabular}{lllll}
\hline \hline Quiz subject & Verbal & Math & Graphical & Pictoral \\
\hline 202 Diffraction, reform & $\mathbf{X}$ & 0.06 & $\mathbf{X}$ & $\mathrm{X}$ \\
202 Spectroscopy, reform & $\mathrm{X}$ & $\mathrm{X}$ & $\mathrm{X}$ & $\mathrm{X}$ \\
\hline \hline
\end{tabular}


An electrostatic air filter uses electric fields to attract charged particles of dust, pollen, etc. to one of two electrically charged metal plates.

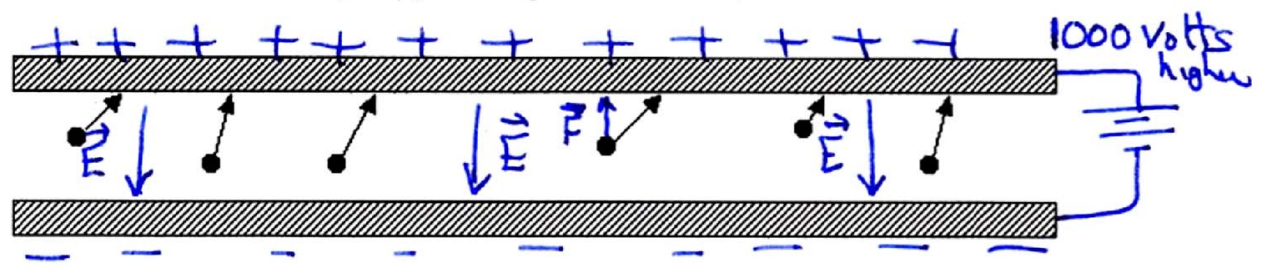

The dust particles are first electrically charged by a small electric discharge and then blown along with the air left to right between the charged plates using a fan. The two plates are wired to a voltage supply so the top plate is 1000 volts higher in voltage than the bottom plate. The two plates are spaced $1 \mathrm{~cm}$ apart. The particles are strongly attracted to the top plate and stick there. They can later be removed by just wiping the top plate with a cloth.

a) How are the particles charged, i.e. are they positive or negative? Explain.
Negatively changed sm e they are attractid to the

b)Indicate on the figure the direction on the electric field between the two plates and determine the magnitude of that electric field.

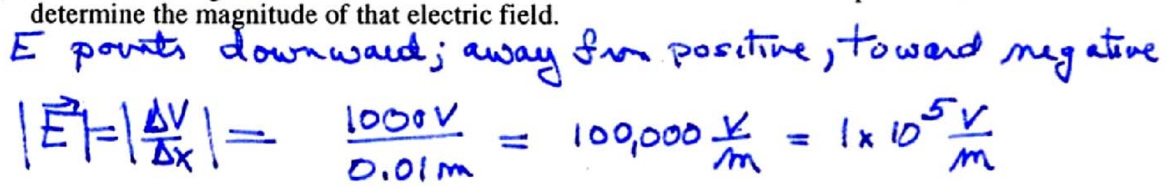

FIG. 3. (Color) Example exam problem (and instructor solution) with pictorial, mathematical, and verbal components. The problem is from a reform 202 exam. combination thereof. The total fraction of the exam composed of a particular representation was defined as the fraction of points possible that came from problems belonging to that representation. Problems with more than one representational component had their points counted in full towards each of the relevant representations; no effort was made to weight the components (for instance, we did not designate a problem as $80 \%$ pictorial and $20 \%$ mathematical, but rather as $100 \%$ of each). Both the problem presentation and the intended solution were considered. For example, a ten-point problem that requires a student to read information off of a provided graph in order to do a numerical calculation is designated mathematical/graphical, and ten points are assigned to both the mathematical and graphical categories. Thus, an exam that is rich in representations can have more than 100 points of representational content assigned to it in this analysis. Any problem that explicitly involves more than one representation has its points counted towards a multiple representations category as well. Once we characterized each exam in terms of its representational content, we calculated the average representational content of the exams in each of the courses. The representational categories are defined as follows.

(i) Verbal: For multiple choice questions, the available answers are phrases that are conceptual or qualitative in nature. For long-answer questions, there is an "Explain your answer" or similar written component.

(ii) Mathematical: The problem requires numerical or algebraic calculation, manipulation, or interpretation, or other significant and explicitly quantitative reasoning.

(iii) Graphical: A graph presents relevant information, or students have to construct a graph. Diagrams that have labeled axes have a graphical component.

(iv) Pictorial: Students must draw or modify a picture, a picture contains needed information, or a picture meaningfully depicts the relevant physical situation. A picture of a
CD accompanying a "plug 'n chug" problem about the wavelength of a CD-reading laser would not fit this standard.

In Fig. 3 we see an example exam problem with solution. Part A of this problem was judged by the above standards to have verbal and pictorial components. Part A was worth 11 points (out of 100), and so there was an 11 point verbal component and an 11 point pictorial component for the sake of the averaging. Part B was judged to have mathematical and pictorial components.

\section{Analysis of long-answer homeworks}

In addition to web-based CAPA problems (generally formulaic in nature for all classes studied here), students were assigned several additional homeworks requiring more indepth work. Here, we consider those from the two 202 classes (traditional and reformed) for the sake of direct com-

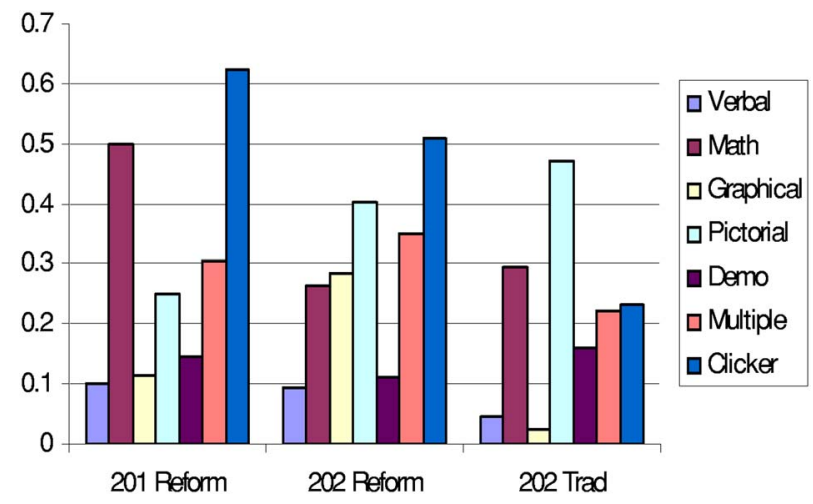

FIG. 4. (Color) Representational content of the lectures for the reformed 201, reformed 202, and traditional 202 courses. "Multiple" category indicates use of multiple representations. Clicker category indicates fraction of class time involving questions that used a personal response system. 


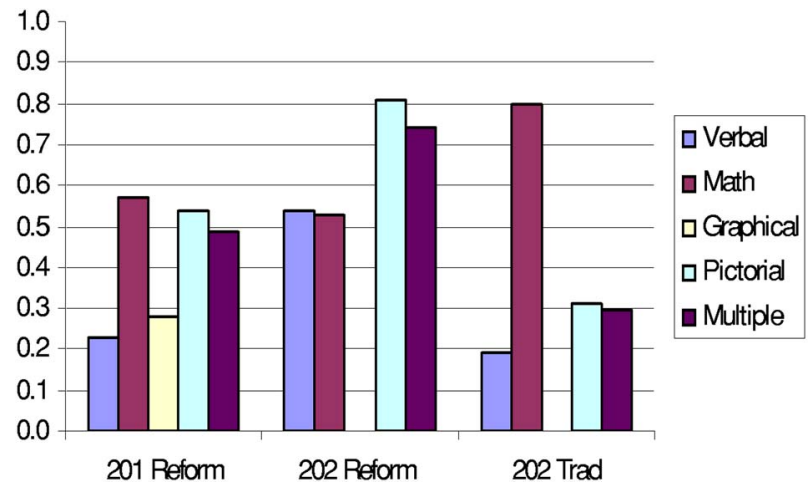

FIG. 5. (Color) Distribution of representations used in the exams in the three courses studied here. Also includes the fraction of exam problems that required explicit use of multiple representations.

parison. We use essentially the same coding scheme as with the exams. The representational content of each assignment is broken down and weighted according to point value. Then, the contents of all assignments are averaged together. The reform 202 course had eight homeworks of this sort, and the traditional 202 had fifteen.

\section{B. Data}

\section{Lecture content}

In Fig. 4, we see the representational content in the reformed Physics 201, reformed 202, and traditional 202 lectures according to the standards described previously. Differences exist between all three sets, suggesting (not surprisingly) that both instructor and content have a significant effect on representation use. That is, a particular instructor will use a different selection of representations in different courses, and in a particular course, different instructors will use different selections of representation. Most relevant to us is the comparison between the reform and traditional sections of 202. The reform section shows a broader selection of representations, with the verbal, math, graphical, and pictorial fractions summing to 1.04 versus 0.83 in the traditional section. We also see more use of multiple representations $(0.35$ versus 0.22 , significant at the $p=0.03$ level using a two-tailed binomial proportion test), and much more use of interactive Clicker questions $(0.51$ versus $0.23, p<0.0001)$. The Clicker category does not describe a representation in itself; rather, it tells us something about how representations are used in the course. The resolution of these data are limited by the interrater reliability (on the order of 0.01 ), and by the division of the lectures into one-minute blocks. Each data set contains three 50-minute lectures, or 150 blocks, suggesting a resulting imprecision on the order of 0.01 .

\section{Exam content}

In Fig. 5, we show the representational content of the exams in the reformed Physics 201, reformed 202, and traditional 202 courses. These data show the average across all exams in each course, excluding the final exam. We see the

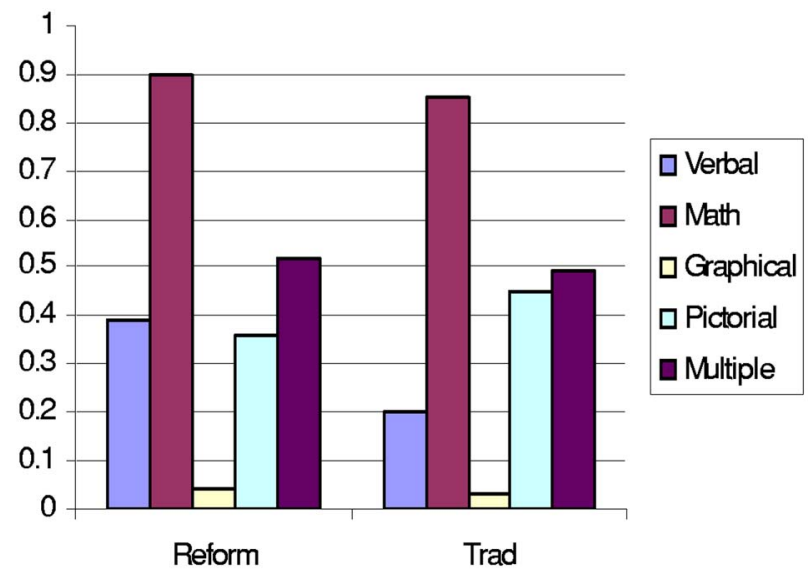

FIG. 6. (Color) Distrubution of representations used in the longformat homeworks in the two Physics 202 courses studied here (reform and traditional). Also includes fraction of the homework assignments that required explicit use of multiple representations.

fraction of the exam problems (weighted according to their point value) that were verbal in nature, mathematical, graphical, and pictorial. We also see the fraction of the exam problems that required explicit use of multiple representations.

It is clear that the exams from the reform sections of 201 and 202 made use of a broader selection of representations than the traditional 202 section. Perhaps most striking is the difference in the proportion of multiple-representations problems, with 0.49 for the reform 201 course and 0.74 for the reform 202 course versus 0.30 for the traditional course. The difference between the reform 202 and traditional 202 figures is statistically significant $(p<0.0001$, two-tailed binomial proportion test).

\section{Homework content}

In Fig. 6 we see the distribution of representations used in the reformed and traditional 202 course homeworks. The distributions are very similar, with the reform course making somewhat greater use of verbal representations and the traditional course making greater use of pictorial representations. The data are similar enough that we can make no claims regarding significant differences in representation quantity.

\section{DISCUSSION}

Our original hypothesis appears to be validated. The reform 202 course shows choice/control performance splits that are much more consistent with the reform 201 data than with the traditional 202 data. We thus conclude that these choice/control splits are associated more with the instructional environment than the content area. The course analysis data demonstrate that major components of the class (in particular, the lectures and exams) were strikingly different in their representational character, with the reform content being richer and using multiple representations more frequently. We also believe that the reform course made more productive use of these representations. For example, half of the reform 202 homeworks require students to perform esti- 
mations as well as straightforward calculations on mathematical problems, while none of the traditional 202 homeworks do so. The way that the course used representations is not described by the data shown here, which only indicate how often categories of representation were used. We will make no attempt to quantify the productivity of representation use in this paper, though we suspect that such a quantification would increase the differences shown (for instance, making a visible difference in the character of the longformat homeworks in each class).

This richer use of representations in-class is consistent with the notion that these students are learning a broader set of representational skills, which could explain the choice/ control splits or lack thereof. With this broader set, working in a chosen representation as opposed to an assigned one has less impact on performance. It is also perhaps significant that the reform 202 section shows generally smaller performance variations across representation within the choice and control groups. Further, this interpretation allows for the fact that the direction of the choice/control splits can vary across topics. Student quiz comments (examined in the previous study) and student interviews not analyzed here show that students have definite opinions regarding their abilities and the relative worth of different problem representations and that these opinions are generally constant across topics. However, it would appear that their problem-solving skills in different representations are not constant across topics, especially in the traditional course. Thus, students are sometimes correct and sometimes incorrect when they assert that they are better with a particular representation. This is in itself something of a metarepresentational failure, though an understandable one, as physics students are not often asked to engage in such metarepresentational tasks. Future work using problemsolving interviews will consider in more detail how accurately students assess their own representational competence.

We do not have evidence to claim that students in the reform sections were necessarily learning better metarepresentional skills than the students in the traditional section. It is quite conceivable that these students were no better than those in the traditional 202 course at assessing their own abilities and evaluating the different representations available to them, but that their broader set of representational skills made any metarepresentational failures less significant. Of course, neither do the data allow us to conclude that the reform 202 students were not learning better metarepresentational skills. To our knowledge, no one has yet undertaken the research task of measuring metarepresentational competence in physics, though some work has characterized student metarepresentational skills. ${ }^{9,11}$

In our first paper on this topic ${ }^{1}$ we suggested that a complete analysis would require attention to both micro-level and macro-level features of the environment. The first paper focused primarily on microscale, specific features, demonstrating how particular problem elements, topics, and representations could affect student performance. In this paper we have taken an explicit look at the effect of instructional environment, considering macrolevel influences that can impact the small scale. For example, instruction can change what resources and skills students bring with them when solving a particular problem. Here, we have inferred that the reform 202 students were more broadly skilled in using different representations. Thus, the students were less vulnerable to particular variations in problem representation. Also, the course analysis presented here demonstrates that the reform course explicitly valued the use of a variety of representations, often together. Such multiple representation use was demonstrated for the students on a regular basis in the lectures, and they were held accountable for such use in their own work, especially on the exams. It is quite likely that this broadscale norm helped drive the development of students' specific representational skills, tying together the different contextual levels $^{21}$ (micro and macro) discussed here.

\section{CONCLUSION}

Our results suggest that instructional environment can play a significant role in developing student representational skills as they apply to problem solving. Pervasive use of different representations and of multiple representations appears to have broadened students' representational skills. Unfortunately, without assessment tasks that are less sensitive to the topic coverage, we are limited in our ability to conclude that student representational skills are stronger in a reformstyle class in an absolute sense; all we are comfortable in asserting is that student problem-solving skills varied significantly less from representation to representation. However, we consider it plausible that as these students develop broader skills, they also develop stronger skills overall. We suspect that if instructors wish to make increased representational facility a primary goal of their course, they can do so effectively so long as their efforts span all (or at least most) aspects of a course.

\section{ACKNOWLEDGMENTS}

This work was supported in part by the NSF (Grant No. REC 0448176), and by the Colorado PhysTEC. Special thanks to the rest of the Physics Education Research group at the University of Colorado at Boulder. Thanks also to Noah Podolefsky and Dr. Beale, Dr. Munsat, and Dr. Peterson for their cooperation and aid.
${ }^{1}$ P. B. Kohl and N. D. Finkelstein, Student representational competence and self-assessment when solving physics problems, Phys. Rev. ST Phys. Educ. Res. 1, 010104 (2005).

${ }^{2}$ D. E. Meltzer, Relation between students' problem-solving per- formance and representational mode, Am. J. Phys. 73, 463 (2005).

${ }^{3}$ R. J. Beichner, Testing student interpretation of kinematics graphs, Am. J. Phys. 62, 750 (1994). 
${ }^{4}$ F. M. Goldberg and J. H. Anderson, Student difficulties with graphical representations of negative values of velocity, Phys. Teach. 27, 254 (1989).

${ }^{5}$ L. C. McDermott, M. L. Rosenquist, and E. H. Van Zee, Student difficulties in connecting graphs and physics: Examples from kinematics, Am. J. Phys. 55, 503 (1987).

${ }^{6}$ A. Van Heuvelen and X. Zou, Multiple representations of workenergy processes, Am. J. Phys. 69, 184 (2001).

${ }^{7}$ A. Van Heuvelen, Learning to think like a physicist: A review of research-based instructional strategies, Am. J. Phys. 59, 891 (1991).

${ }^{8}$ R. J. Dufresne, W. J. Gerace, and W. J. Leonard, Solving physics problems with multiple representations, Phys. Teach. 35, 270 (1997).

${ }^{9}$ A. A. diSessa and B. L. Sherin, Meta-representation: an introduction, J. Math. Behav. 19, 385 (2000).

${ }^{10}$ A. A. diSessa, D. Hammer, B. L. Sherin, and T. Kolpakowski, Inventing graphing: Meta-representational expertise in children, J. Math. Behav. 10, 117 (1991).

${ }^{11}$ A. A. diSessa, Students' criteria for representational adequacy, in Symbolizing, Modeling and Tool Use in Mathematics Education, edited by K. Gravemeijer, R. Lehrer, B. van Oers, and L. Vershaffel (Kluwer Academic Publishers, Dordrecht, The Netherlands, 2003).

${ }^{12} \mathrm{~F}$. Reif, Understanding and teaching important scientific thought processes, Am. J. Phys. 63, 17 (1995).

${ }^{13}$ D. Giancoli, Physics, 5th ed. (Prentice-Hall, Upper Saddle River, NJ, 1998).

${ }^{14}$ E. Mazur, Peer Instruction: A User's Manual (Prentice-Hall, Upper Saddle River, NJ, 1996).

${ }^{15}$ http://www.h-itt. com/ (retrieved July 4, 2005).

${ }^{16} \mathrm{~K}$. R. Koedinger and M. J. Nathan, The real story behind story problems: Effects of representations on quantitative reasoning, J. Learn. Sci. 13, 129 (2004).

${ }^{17}$ Note that the students in the reformed 202 section were largely those from the reformed 201 section. The students in the traditional 202 course had, for the most part, taken a traditional 201 course as well. Thus, when we compare the two 202 sections, we should be aware that any significant differences may be the cumulative result of two semesters of instruction, not one.

${ }^{18}$ One potential weakness in our data stems from the fact that if student absolute performance is too low, relative performances will necessarily be very even regardless of student representational skill. However, only one of the four reform course quizzes [two in 202 presented here and two in 201 (Ref. 11)] shows very low absolute numbers, and these are not present if one considers the likely student misinterpretation described above. Thus, we are confident that the lack of choice/control splits in the reform courses is a genuine effect.

${ }^{19}$ The LearningOnline network with capa, http://www.lon-capa.org (retrieved July 4, 2005).

${ }^{20}$ We chose one-minute intervals as that seemed a reasonable order of magnitude for discrete portions of a discussion or explanation. Intervals on the order of several seconds result in all intervals containing single representations. Intervals of several minutes tend to include all or nearly all possible representations. As a check, we repeated the analysis with two-minute intervals. The figures for multiple representational use were modestly increased, the other categories increased less, and the relative class-to-class variations (which are of the most interest here) were essentially unchanged.

${ }^{21}$ N. D. Finkelstein, Learning physics in context: A study of student learning about electricity and magnetism, Int. J. Sci. Educ. 27, 1187 (2005). 